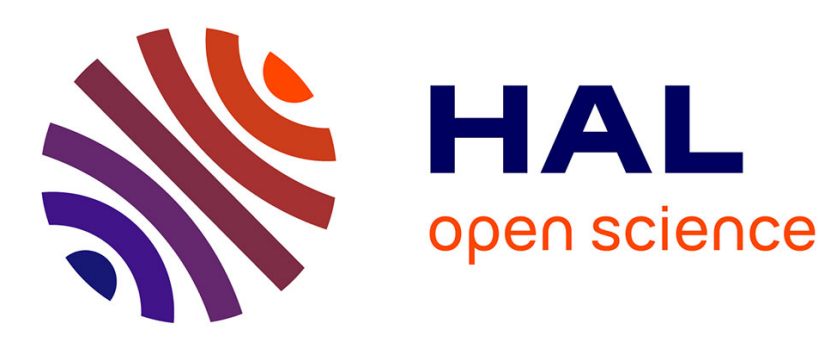

\title{
Mixed Integer Nonlinear Optimization Models for the Euclidean Steiner Tree Problem in R
}

\author{
Hacène Ouzia, Nelson Maculan
}

\section{To cite this version:}

Hacène Ouzia, Nelson Maculan. Mixed Integer Nonlinear Optimization Models for the Euclidean Steiner Tree Problem in R. Journal of Global Optimization, 2021, 10.1007/s10898-021-01001-6 . hal02293105v2

\section{HAL Id: hal-02293105 \\ https://hal.sorbonne-universite.fr/hal-02293105v2}

Submitted on 23 Sep 2019

HAL is a multi-disciplinary open access archive for the deposit and dissemination of scientific research documents, whether they are published or not. The documents may come from teaching and research institutions in France or abroad, or from public or private research centers.
L'archive ouverte pluridisciplinaire $\mathbf{H A L}$, est destinée au dépôt et à la diffusion de documents scientifiques de niveau recherche, publiés ou non, émanant des établissements d'enseignement et de recherche français ou étrangers, des laboratoires publics ou privés. 


\title{
Mixed Integer Nonlinear Optimization Models for the Euclidean Steiner Tree Problem in $\mathbb{R}^{d}$
}

\author{
Hacene Ouzia* \\ Sorbonne Universite, CNRS, LIP-6, F-75005, Paris, France \\ Nelson Maculan \\ Universidade Federal do Rio de Janeiro, COPPE \& IM, 21941-972 Rio de Janeiro, Brazil
}

\begin{abstract}
New mixed integer nonlinear optimization models for the Euclidean Steiner tree problem in $d$-space (with $d \geq 3$ ) will be presented in this work. Each model features a non smooth objective function but a convex set of feasible solutions. All these models are theoretically equivalent. From these models, six mixed integer linear and nonlinear relaxations will be considered. Each relaxation has the same set of feasible solutions as the model from which it is derived. Finally, preliminary computational results highlighting the main features of the presented relaxations will be discussed.
\end{abstract}

Keywords: Integer Programming, Euclidean Steiner tree problem, Steiner tree, Nonlinear optimization models, Mixed integer nonlinear optimization,

Relaxation.

\section{Introduction}

Given $n$ points in $\mathbb{R}^{d}$ (called terminals) the goal in the Euclidean Steiner Tree Problem (ESTP) is to find the $\|\cdot\|_{2}$-shortest tree that spans these points using or not extra points (called Steiner points). The length of each edge of the tree is the Euclidean distance between its ends. A very important history of the (ESTP) can be found in [1].

The Euclidean Steiner tree problem is an NP-hard optimization problem $($ see $[13,12])$. It has several applications, to cite a few: phylogenetics (see [16, 3, $2])$ and structure and folding proteins (see $[19,17])$. In this work, the dimension $d$ will be assumed at least equal to 3 (for the case $d=2$ see $[20,5,4]$ ).

As explained in [8], exact approaches to the (ESTP) can be divided into two categories. The approaches in the first category are enumeration based

\footnotetext{
*Corresponding author

Email addresses: hacene.ouzia@sorbonne-universite.fr (Hacene Ouzia), maculan@cos.ufrj.br (Nelson Maculan)
} 
approaches. The first enumeration based approaches are the two algorithms proposed by Gilbert and Pollak in [14] and Smith in [18]. The approaches of the second category are based on a mathematical model of the (ESTP). The first of these mathematical models is the mixed integer nonlinear optimization formulation presented in [15]. From this formulation two other formulations of the (ESTP) can be found in [9] and [10] (see [8] for more details). More recent exact approaches can be found in $[11,6,9,10,7]$.

In this work, new mixed integer nonlinear models for the Euclidean Steiner tree problem will be presented. All these models are derived from the model given in [15], and are all theoretically equivalent. But, from a computational point of view they are different. Also, all the models feature a non smooth objective function. From these models several mixed integer linear and nonlinear relaxations will be considered. Each relaxation has a smooth objective function which lower bounds the objective function of the model from which it is derived and it has the same set of feasible solutions as the set of feasible solutions of the model from which it is derived. Solving these relaxations using an ad hoc optimization solver leads to a lower and upper bounds of an optimal solution of the Euclidean Steiner tree problem.

The paper is organized as follows. In the next section, the mixed integer nonlinear optimization models of the (ESTP) given in [15] and [10] will be recalled. In section 3, new mixed integer nonlinear optimization models for the (ESTP) will be presented. In section 4, six mixed integer linear and nonlinear relaxations of the (ESTP) will be considered. Preliminary computational results will be presented in section 5 . Concluding remarks will be given in the final section.

\section{Previous Models}

In this section, the mixed integer nonlinear formulations of the Euclidean Steiner tree problem given in [15] and [10] will be recalled.

To simplify the presentation, some notations and definitions are necessary. Let $P$ equal to $\{1, \ldots, n\}$ the index set the terminals $\zeta^{1}, \zeta^{2}, \ldots, \zeta^{n}$ and let $X$ equal to $\{1, \ldots, n-2\}$ the index set of the Steiner points $x^{1}, x^{2}, \ldots, x^{n-2}$. The terminals and Steiner points all belong to $\mathbb{R}^{d}$.

A Steiner tree is any spanning tree connecting the $n$ terminals $\zeta^{1}, \zeta^{2}, \ldots, \zeta^{n}$, having at least one Steiner point, and the coordinates of its Steiner points are all known. If the coordinates of the Steiner points of a Steiner tree are not fixed then the resulting tree is called the topology of the Steiner tree. A topology is called a Steiner topology if the degree of any Steiner point is 3 and the degree of any terminal is at most 3 . In the case where the number of Steiner points in a Steiner topology equals $n-2$ then this topology is called a full Steiner topology. 


\subsection{Formulation by Maculan, Michelon and Xavier}

In the formulation by Maculan, Michelon and Xavier (see [15]) the Euclidean Steiner tree problem is modeled as follows:

$$
\begin{array}{ll}
\min & \sum_{p \in P, q \in X} y_{p q}\left\|x^{q}-\zeta^{p}\right\|_{2}+\sum_{p, q \in X: p<q} z_{p q}\left\|x^{q}-x^{p}\right\|_{2} \\
\text { s.t. } & \\
& \sum_{q \in X} y_{p q}=1, p \in P, \\
& \sum_{p \in P} y_{p q}+\sum_{p \in X: p<q} z_{p q}+\sum_{p \in X: p>q} z_{q p}=3, q \in X, \\
& \sum_{p \in X: p<q} z_{p q}=1, q \in X, q>1, \\
& \sum_{p \in P} y_{p q} \leq 2, q \in X, \\
& x^{p} \in \mathbb{R}^{d}, p \in P, \\
& y_{p q} \in\{0,1\}, p \in P, q \in X, \\
& z_{p q} \in\{0,1\}, p \in X, q \in X, p<q,
\end{array}
$$

where $\|\cdot\|_{2}$ is the Euclidean norm in $\mathbb{R}^{d}$. The sum over an empty set is equal, by convention, to zero.

In a feasible solution $(y, z)$ satisfying the constraints (2)-(8) the variable $y_{p q}$ equals 1 if and only if the terminal $\zeta^{p}$ is connected to the Steiner point $x^{q}$ and the variable $z_{p q}$ equals 1 if and only if the two Steiner points $x^{p}$ and $x^{q}$ are connected together. As shown in [15], to any feasible solution $(y, z)$ corresponds a unique Steiner tree having a full topology. Any optimal solution of the optimization problem (1)-(8) is called a minimal Steiner tree.

Regarding the constraints (2)-(8): constraints (2) indicate that the degree of a terminal is equal to 1 ; constraints (3) indicate that the degree of a Steiner point is equal to 3; and constraints (4) eliminate subtours among Steiner points. Finally, constraints (5) are only used to strengthen the model and notice that they are not necessarily valid in the plane.

In the sequel it will be assumed that:

Assumption 1. For any indexes $p$ and $q$ that belong to $P$ with $p<q$ :

$$
\left\|\zeta^{p}-\zeta^{q}\right\|_{\infty} \leq \frac{1}{d} \text { and }\left\|\zeta^{p}\right\|_{\infty} \leq 1
$$

Notice that this assumption is not restrictive. Indeed, if

$$
\max \left\{\left\|\zeta^{p}-\zeta^{q}\right\|_{\infty}: p, q \in P\right\}=\delta \text { and } \max \left\{\left\|\zeta^{p}\right\|_{\infty}: p \in P\right\}=\beta,
$$

where $\delta>\frac{1}{d}$ and $\beta>1$, then the assumption will be fulfilled if the coordinates of the terminals are divided by the factor $\delta \beta d$. 


\subsection{Formulation by Fampa and Maculan}

The formulation by Fampa and Maculan (see [9] and [10]) is derived from the formulation by Maculan, Michelon and Xavier (see [15]) as follows.

Using the following substitutions:

$$
\begin{aligned}
& y_{p q}\left\|x^{q}-\zeta^{p}\right\|_{2}=w_{p q}, p \in P, q \in X, \\
& z_{p q}\left\|x^{q}-x^{p}\right\|_{2}=v_{p q}, p, q \in X, p<q,
\end{aligned}
$$

in the objective function and adding only the following valid constraints to the set of feasible solutions (2)-(8):

$$
\begin{array}{r}
w_{p q} \geq\left\|x^{q}-\zeta^{p}\right\|_{2}+y_{p q}-1, p \in P, q \in X, \\
v_{p q} \geq\left\|x^{q}-x^{p}\right\|_{2}+z_{p q}-1, p, q \in X, p<q, \\
w_{p q} \geq 0, p \in P, q \in X, \\
v_{p q} \geq 0, p \in X, q \in X, p<q .
\end{array}
$$

Indeed, for any indexes $p$ and $q$ that belong to $P$, if in an optimal solution the variable $y_{p q}$ is equal to 0 then the corresponding constraint in (9) implies that:

$$
w_{p q} \geq\left\|x^{q}-\zeta^{p}\right\|_{2}-1 \text {. }
$$

According to the assumption 1 and the corresponding constraint in (11) it follows that $w_{p q}$ is non negative and since we are minimizing its value in an optimum solution is 0 . Now, if $y_{p q}$ is equal to 1 then the corresponding constraint in (9) implies that:

$$
w_{p q} \geq\left\|x^{q}-\zeta^{p}\right\|_{2} .
$$

Again, since we are minimizing then at the optimum this last constraint must be satisfied at equality. The same arguments apply to the other constraints.

Thus, one ends up with the following formulation:

$$
\min _{p \in P, q \in X} w_{p q}+\sum_{p \in X, q \in X: p<q} v_{p q}
$$$$
(2)-(8) \text { and }(9)-(12) \text {. }
$$

In the sequel, the same technique will be applied to derive new mixed integer nonlinear optimization models for the Euclidean Steiner tree problem.

\section{New Models}

In this section, new models for the Euclidean Steiner tree problem will be presented. These models, as stated before, are all equivalent and derived from the model in [15]. In these models, due to the presence of the square root, only the objective function is non convex et non smooth. In the next section, several relaxations will be derived from these models.

Remark 2. In what follows, the fact that the variables $y$ and $z$ are binary will be used frequently without mentioning it explicitly. 


\subsection{First Model}

Notice that the objective function (1) can be written as follows:

$$
\sum_{p \in P, q \in X} \sqrt{\sum_{j=1}^{d}\left[y_{p q}\left(x_{j}^{q}-\zeta_{j}^{p}\right)\right]^{2}}+\sum_{p, q \in X: p<q} \sqrt{\sum_{j=1}^{d}\left[z_{p q}\left(x_{j}^{q}-x_{j}^{p}\right)\right]^{2}} .
$$

Notice that we used the fact that $y_{p q}$ and $z_{p q}$ are equal to $y_{p q}^{2}$ and $z_{p q}^{2}$, respectively. Using the following substitutions:

$$
\begin{gathered}
v_{p q}^{j}=y_{p q}\left(x_{j}^{q}-\zeta_{j}^{p}\right), p \in P, q \in X, j \in\{1, \ldots, d\}, \\
w_{p q}^{j}=z_{p q}\left(x_{j}^{q}-x_{j}^{p}\right), p, q \in X, p<q, j \in\{1, \ldots, d\},
\end{gathered}
$$

the objective function (1) reads:

$$
\sum_{p \in P, q \in X} \sqrt{\sum_{j=1}^{d}\left(v_{p q}^{j}\right)^{2}}+\sum_{p, q \in X: p<q} \sqrt{\sum_{j=1}^{d}\left(w_{p q}^{j}\right)^{2}} .
$$

The following constraints can be added to strengthen the set (2)-(8):

$$
\begin{aligned}
-y_{p q} & \leq v_{p q}^{j} \leq y_{p q}, p \in P, q \in X, j \in\{1, \ldots, d\}, \\
-z_{p q} & \leq w_{p q}^{j} \leq z_{p q}, p, q \in X, p<q, j \in\{1, \ldots, d\}, \\
v_{p q}^{j} & \geq y_{p q}-1+\left(x_{j}^{q}-\zeta_{j}^{p}\right), p \in P, q \in X, j \in\{1, \ldots, d\}, \\
v_{p q}^{j} & \leq\left(x_{j}^{q}-\zeta_{j}^{p}\right)+1-y_{p q}, p \in P, q \in X, j \in\{1, \ldots, d\}, \\
w_{p q}^{j} & \geq z_{p q}-1+\left(x_{j}^{q}-x_{j}^{p}\right), p, q \in X, p<q, j \in\{1, \ldots, d\}, \\
w_{p q}^{j} & \leq\left(x_{j}^{q}-x_{j}^{p}\right)+1-z_{p q}, p, q \in X, p<q, j \in\{1, \ldots, d\} .
\end{aligned}
$$

So, the resulting model reads:

$$
\begin{aligned}
& \min \sum_{p \in P, q \in X} \sqrt{\sum_{j=1}^{d}\left(v_{p q}^{j}\right)^{2}}+\sum_{p, q \in X: p<q} \sqrt{\sum_{j=1}^{d}\left(w_{p q}^{j}\right)^{2}} \\
& \text { s.t. } \\
& \quad(2)-(8) \text { and }(17)-(22) .
\end{aligned}
$$

\subsection{Second Model}

The objective function (1) can be written differently using the following substitutions:

$$
v_{p q}=\sum_{j=1}^{d} y_{p q}\left(x_{j}^{q}-\zeta_{j}^{p}\right)^{2}, p \in P, q \in X
$$




$$
w_{p q}=\sum_{j=1}^{d} z_{p q}\left(x_{j}^{q}-x_{j}^{p}\right)^{2}, p, q \in X, p<q
$$

Then, the following constraints can be used instead to strengthen the set (2)-(8).

$$
\begin{array}{r}
0 \leq v_{p q} \leq y_{p q}, p \in P, q \in X, \\
0 \leq w_{p q} \leq z_{p q}, p, q \in X, p<q, \\
v_{p q} \geq y_{p q}-1+\sum_{j=1}^{d}\left(x_{j}^{q}-\zeta_{j}^{p}\right)^{2}, p \in P, q \in X, \\
v_{p q} \leq \sum_{j=1}^{d}\left(x_{j}^{q}-\zeta_{j}^{p}\right)^{2}, p \in P, q \in X, \\
w_{p q} \geq z_{p q}-1+\sum_{j=1}^{d}\left(x_{j}^{q}-x_{j}^{p}\right)^{2}, p, q \in X, p<q, \\
w_{p q} \leq 1-z_{p q}+\sum_{j=1}^{d}\left(x_{j}^{q}-x_{j}^{p}\right)^{2}, p, q \in X, p<q .
\end{array}
$$

This leads to the following second model:

$$
\begin{aligned}
& \min \sum_{p \in P, q \in X} \sqrt{v_{p q}}+\sum_{p, q \in X: p<q} \sqrt{w_{p q}} \\
& \text { s.t. } \\
& \quad(2)-(8) \text { and }(25)-(30) .
\end{aligned}
$$

\subsection{Third Model}

This third model is based on a different expression of the objective function (1). Indeed, it follows using the following substitutions:

$$
u_{p q}^{j}=y_{p q} x_{j}^{q}, p \in P, q \in X, j \in\{1, \ldots, d\},
$$

that:

$$
\sum_{j=1}^{d} y_{p q}\left(x_{j}^{q}-\zeta_{j}^{p}\right)^{2}=\sum_{j=1}^{d}\left(u_{p q}^{j}-\zeta_{j}^{p} y_{p q}\right)^{2} .
$$

And, using these other substitutions:

$$
\begin{gathered}
v_{p q}^{j}=z_{p q} x_{j}^{p}, p, q \in X, p<q, j \in\{1, \ldots, d\}, \\
w_{p q}^{j}=z_{p q} x_{j}^{q}, p, q \in X, p<q, j \in\{1, \ldots, d\},
\end{gathered}
$$


it follows that:

$$
\sum_{j=1}^{d} z_{p q}\left(x_{j}^{q}-x_{j}^{p}\right)^{2}=\sum_{j=1}^{d}\left(w_{p q}^{j}\right)^{2}-\sum_{j=1}^{d} x_{j}^{p} w_{p q}^{j}-\sum_{j=1}^{d} x_{j}^{q} v_{p q}^{j}+\sum_{j=1}^{d}\left(v_{p q}^{j}\right)^{2} .
$$

Thus, one can add the following constraints:

$$
\begin{gathered}
-y_{p q} \leq u_{p q}^{j} \leq y_{p q}, p \in P q \in X, j \in\{1, \ldots, d\}, \\
u_{p q}^{j} \leq x_{j}^{q}+1-y_{p q}, p \in P, q \in X, j \in\{1, \ldots, d\}, \\
u_{p q}^{j} \geq x_{p}^{q}-1+y_{p q}, p \in P, q \in X, j \in\{1, \ldots, d\},
\end{gathered}
$$

and

$$
\begin{gathered}
-z_{p q} \leq v_{p q}^{j} \leq z_{p q}, p, q \in X, p<q, j \in\{1, \ldots, d\}, \\
v_{p q}^{j} \leq x_{j}^{p}+1-z_{p q}, p, q \in X, p<q, j \in\{1, \ldots, d\}, \\
v_{p q}^{j} \geq x_{j}^{p}-1+z_{p q}, p, q \in X, p<q, j \in\{1, \ldots, d\}, \\
w_{p q}^{j} \leq z_{p q}, p, q \in X, p<q, j \in\{1, \ldots, d\}, \\
w_{p q}^{j} \geq-z_{p q}, p, q \in X, p<q, j \in\{1, \ldots, d\}, \\
w_{p q}^{j} \leq x_{j}^{q}+1-z_{p q}, p, q \in X, p<q, j \in\{1, \ldots, d\}, \\
w_{p q}^{j} \geq x_{j}^{q}-1+z_{p q}, p, q \in X, p<q, j \in\{1, \ldots, d\},
\end{gathered}
$$

to strengthen the set (2)-(8). Then, one ends up with the following third model:

$$
\begin{aligned}
& \min \sum_{p \in P, q \in X} \sqrt{\sum_{j=1}^{d}\left(u_{p q}^{j}-\zeta_{j}^{p} y_{p q}\right)^{2}}+ \\
& \sum_{p, q \in X: p<q} \sqrt{\left(\sum_{j=1}^{d}\left(w_{p q}^{j}\right)^{2}-\sum_{j=1}^{d} x_{j}^{p} w_{p q}^{j}-\sum_{j=1}^{d} x_{j}^{q} v_{p q}^{j}+\sum_{j=1}^{d}\left(v_{p q}^{j}\right)^{2}\right)} \\
& \text { s.t. } \\
& \quad(2)-(8) \text { and }(36)-(45) .
\end{aligned}
$$

\subsection{Fourth Model}

Considering the same substitutions (31), (33) and (34) as in the previous model it follows that:

$$
\sum_{j=1}^{d} y_{p q}\left(x_{j}^{q}-\zeta_{j}^{p}\right)^{2}=\sum_{j=1}^{d}\left(u_{p q}^{j}-\zeta_{j}^{p} y_{p q}\right)^{2},
$$


and

$$
\sum_{j=1}^{d} z_{p q}\left(x_{j}^{q}-x_{j}^{p}\right)^{2}=\sum_{j=1}^{d}\left(w_{p q}^{j}-v_{p q}^{j}\right)^{2} .
$$

Now, one can add the following constraints:

$$
\begin{gathered}
-y_{p q} \leq u_{p q}^{j} \leq y_{p q}, p \in P, q \in X, j \in\{1, \ldots, d\}, \\
u_{p q}^{j} \leq x_{j}^{q}+1-y_{p q}, p \in P, q \in X, j \in\{1, \ldots, d\}, \\
u_{p q}^{j} \geq x_{j}^{q}-1+y_{p q}, p \in P, q \in X, j \in\{1, \ldots, d\},
\end{gathered}
$$

and

$$
\begin{aligned}
-z_{p q} & \leq v_{p q}^{j} \leq z_{p q}, p, q \in X, p<q, j \in\{1, \ldots, d\}, \\
v_{p q}^{j} & \leq x_{j}^{p}+1-z_{p q}, p, q \in X, p<q, j \in\{1, \ldots, d\}, \\
v_{p q}^{j} & \geq x_{j}^{p}-1+z_{p q}, p, q \in X, p<q, j \in\{1, \ldots, d\}, \\
w_{p q}^{j} & \leq z_{p q}, p, q \in X, p<q, j \in\{1, \ldots, d\}, \\
w_{p q}^{j} & \geq-z_{p q}, p, q \in X, p<q, j \in\{1, \ldots, d\}, \\
w_{p q}^{j} & \leq x_{j}^{q}+1-z_{p q}, p, q \in X, p<q, j \in\{1, \ldots, d\}, \\
w_{p q}^{j} & \geq x_{j}^{q}-1+z_{p q}, p, q \in X, p<q, j \in\{1, \ldots, d\},
\end{aligned}
$$

to strengthen the set (2)-(8). Then, one ends up with the following model:

$$
\min \sum_{p \in P, q \in X} \sqrt{\sum_{j=1}^{d}\left(u_{p q}^{j}-\zeta_{j}^{p} y_{p q}\right)^{2}}+\sum_{p, q \in X: p<q} \sqrt{\sum_{j=1}^{d}\left(w_{p q}^{j}-v_{p q}^{j}\right)^{2}}
$$

s.t.

$$
\text { (2) - (8) and (48) - (57). }
$$

\subsection{Equivalence of the Four Models}

All the previous models are equivalent, that is for any optimal solution of one model, these exists an optimal solution of the other having the same objective value. To prove this fact, the main argument is that the variables $y$ and $z$ are kept binary in all these models.

Let us consider the equivalence of the first and fourth models. The other equivalences can be obtained using the same arguments.

Let $X=(x, y, z, u, v, w)$ be an optimal solution of the model $\left(M_{4}\right)$. Consider the following point (of an appropriate dimension) $A=(a, b, c, r, s)$ obtained from $X$ as follows:

$$
a=x, b=y, c=z,
$$




$$
\begin{aligned}
& r_{p q}^{j}=u_{p q}^{j}-y_{p q} \zeta^{p}, p \in P, q \in X, j \in\{1, \ldots, d\}, \\
& s_{p q}^{j}=v_{p q}^{j}-w_{p q}^{j}, p, q \in X, j \in\{1, \ldots, d\} .
\end{aligned}
$$

It is clear that $(b, c)$ satisfies the constraints (2)-(8). Also, since the variables $y$ and $z$ are binary then:

$$
\begin{aligned}
& r_{p q}^{j}=y_{p q}\left(x_{j}^{q}-\zeta^{p}\right)=b_{p q}\left(a_{j}^{q}-\zeta^{p}\right), p \in P, q \in X, j \in\{1, \ldots, d\}, \\
& s_{p q}^{j}=w_{p q}^{j}-v_{p q}^{j}=c_{p q}\left(a_{j}^{q}-a_{j}^{p}\right), p, q \in X, j \in\{1, \ldots, d\},
\end{aligned}
$$

which means that the vector $(a, b, c, r, s)$ satisfies the equalities (14) and (15). Also, notice that:

$$
f_{4}(X)=f_{1}(A),
$$

where $f_{1}$ and $f_{4}$ are the objective functions of the models $\left(M_{1}\right)$ and $\left(M_{4}\right)$, respectively.

Now, assume that the point $A$ is not an optimal solution of the model $\left(M_{1}\right)$. That is, there exists a feasible points $\hat{A}$ of $\left(M_{1}\right)$ such that $f_{1}(A)>f_{1}(\hat{A})$. Let $\hat{A}$ be equal to $(\hat{a}, \hat{b}, \hat{c}, \hat{r}, \hat{s})$. The point $\hat{X}$ defined as follows:

$$
\begin{aligned}
\hat{x} & =\hat{a}, \hat{y}=\hat{b}, \hat{z}=\hat{c}, \\
\hat{u}_{p q}^{j} & =\hat{b}_{p q} \hat{a}_{j}^{q}, p \in P, q \in X, j \in\{1, \ldots, d\}, \\
\hat{v}_{p q}^{j} & =\hat{c}_{p q} \hat{a}_{j}^{p}, p, q \in X, p<q, j \in\{1, \ldots, d\}, \\
\hat{w}_{p q}^{j} & =\hat{c}_{p q} \hat{a}_{j}^{q}, p, q \in X, p<q, j \in\{1, \ldots, d\},
\end{aligned}
$$

is a feasible solution of the model $\left(M_{4}\right)$ and $f_{1}(\hat{A})=f_{4}(\hat{X})$. Thus,

$$
\begin{aligned}
f_{1}(\hat{A}) & =f_{4}(\hat{X}), \\
& \geq f_{4}(X) .
\end{aligned}
$$

Consequently,

$$
f_{1}(\hat{A}) \geq f_{1}(A),
$$

which is a contradiction. Thus, there exists for any optimal solution of the model $\left(M_{4}\right)$ an optimal solution of the model $\left(M_{1}\right)$ with the same objective value. The reverse property can be shown using same arguments.

Despite the theoretical equivalence of these models, from a computational point a view they lead to a non equivalent relaxations.

\section{Relaxations}

In the previous section, four non convex and non smooth mixed integer models for the Euclidean Steiner tree problems were presented. In this section, 
mixed integer linear and nonlinear relaxations will be derived from these models. Each relaxation has the same set of feasible solutions as the set of feasible solutions of the model from which it is derived.

Before that, recall that an optimization problem:

$$
\min \{f(x, y, z):(x, y, z) \in \Omega\},
$$

is a relaxation of the problem (1)-(8) if:

1. the objective function satisfies the following condition:

$$
f(x, y, z) \leq \sum_{p \in P, q \in X} y_{p q}\left\|x^{q}-\zeta^{p}\right\|_{2}+\sum_{p \in X, q \in X: p<q} z_{p q}\left\|x^{q}-x^{p}\right\|_{2},
$$

2. the constraint set $\Omega$ contains the set defined by (2)-(8).

Given in the next proposition, a sufficient condition that guarantees (62) for an objective function $f$ obtained from (1) when the square root in the expression that defining it is ignored.

Proposition 3. If for any indexes $p$ and $q$ that belong to $P$ with $p<q$ such that:

$$
\left\|\zeta^{p}-\zeta^{q}\right\|_{\infty} \leq \frac{1}{d}
$$

then

$$
\begin{aligned}
\sum_{p \in P, q \in X} y_{p q}\left\|x^{q}-\zeta^{p}\right\|_{2}^{2} & +\sum_{p \in X, q \in X: p<q} z_{p q}\left\|x^{q}-x^{p}\right\|_{2}^{2} \\
& \leq \sum_{p \in P, q \in X} y_{p q}\left\|x^{q}-\zeta^{p}\right\|_{2}+\sum_{p \in X, q \in X: p<q} z_{p q}\left\|x^{q}-x^{p}\right\|_{2} .
\end{aligned}
$$

Proof. It is sufficient to notice that, for every $p \in P$ and $q \in X$ :

$$
\begin{aligned}
\left\|x^{q}-\zeta^{p}\right\|_{2}^{2} & \leq d \max _{p \in P, q \in X}\left\{\left\|x^{q}-\zeta^{p}\right\|_{\infty}^{2}\right\}, \\
& \leq d \max _{p, q \in P: p<q}\left\{\left\|\zeta^{q}-\zeta^{p}\right\|_{\infty}^{2}\right\} \leq 1,
\end{aligned}
$$

(all the Steiner points belong to the convex hull of the terminals), for every $p \in X$ and $q \in X$ such that $p<q$ :

$$
\begin{aligned}
\left\|x^{p}-x^{q}\right\|_{2}^{2} & \leq d \max _{p, q \in X: p<q}\left\{\left\|x^{p}-x^{q}\right\|_{\infty}^{2}\right\}, \\
& \leq d \max _{p, q \in P: p<q}\left\{\left\|\zeta^{p}-\zeta^{q}\right\|_{\infty}^{2}\right\} \leq 1,
\end{aligned}
$$

and, finally, any real $x$ that belong to the interval $[0,1]$ is less than its square $\operatorname{root} \sqrt{x}$. This completes the proof. 


\subsection{First Relaxation}

The first relaxation is derived from the model (1)-(8) and it is defined as follows.

$$
\min _{p \in P, q \in X} y_{p q} \sum_{j=1}^{d}\left(x_{j}^{q}-\zeta_{j}^{p}\right)^{2}+\sum_{p \in X, q \in X: p<q} z_{p q} \sum_{j=1}^{d}\left(x_{j}^{q}-x_{j}^{p}\right)^{2}
$$

$(2)-(8)$

The objective function of the relaxation $\left(R_{1}\right)$ is smooth but not convex.

\subsection{Second Relaxation}

The second relaxation is derived from the first model $\left(M_{1}\right)$. It is defined as follows:

$$
\begin{aligned}
& \min _{p \in P, q \in X} \sum_{j=1}^{d}\left(v_{p q}^{j}\right)^{2}+\sum_{p \in X, q \in X: p<q} \sum_{j=1}^{d}\left(w_{p q}^{j}\right)^{2} \\
& \text { s.t. } \\
& \quad(2)-(8) \text { and }(17)-(22) .
\end{aligned}
$$

The relaxation $\left(R_{2}\right)$ has a strict convex quadratic and smooth objective function and its set of feasible solutions is a polyhedron.

\subsection{Third Relaxation}

The third relaxation is derived from the second model $\left(M_{2}\right)$. It is defined as follows:

$$
\begin{aligned}
& \min \sum_{p \in P, q \in X} v_{p q}+\sum_{p, q \in X: p<q} w_{p q} \\
& \text { s.t. } \\
& \quad(2)-(8) \text { and }(25)-(30) .
\end{aligned}
$$

The relaxation $\left(R_{3}\right)$ is a mixed integer nonlinear optimization problem.

\subsection{Fourth Relaxation}

The fourth relaxation is derived from the model $\left(M_{1}\right)$ as follows. Let us set:

$$
\begin{aligned}
& r_{p q}=\left\|v_{p q}\right\|_{\infty}, p \in P, q \in X, \\
& s_{p q}=\left\|w_{p q}\right\|_{\infty}, p, q \in X, p<q .
\end{aligned}
$$

where the variables $v$ and $w$ are defined by (14)-(15).

Noticing the following valid constraints:

$$
r_{p q} \geq v_{p q}^{j}, p \in P, q \in X, j=1, \ldots, d,
$$




$$
\begin{aligned}
& r_{p q} \geq-v_{p q}^{j}, p \in P, q \in X, j=1, \ldots, d, \\
& r_{p q} \geq 0, p \in P, q \in X, \\
& s_{p q} \geq w_{p q}^{j}, p, q \in X, p<q, j=1, \ldots, d, \\
& s_{p q} \geq-w_{p q}^{j}, p, q \in X, p<q, j=1, \ldots, d, \\
& s_{p q} \geq 0, p, q \in X, p<q,
\end{aligned}
$$

and the following fact:

$$
\sum_{p \in P, q \in X} r_{p q}+\sum_{p, q \in X: p<q} s_{p q} \leq \sum_{p \in P, q \in X} y_{p q}\left\|x^{q}-\zeta^{p}\right\|_{2}+\sum_{p \in X, q \in X: p<q} z_{p q}\left\|x^{q}-x^{p}\right\|_{2},
$$

one can consider the following relaxation:

$$
\begin{aligned}
& \min \sum_{p \in P, q \in X} r_{p q}+\sum_{p, q \in X: p<q} s_{p q} \\
& \text { s.t. } \\
& \qquad(2)-(8),(17)-(22) \text { and }(63)-(68) .
\end{aligned}
$$

The relaxation $\left(R_{4}\right)$ is a mixed integer linear optimization problem. Finally, notice that:

$$
\sum_{p \in P, q \in X} r_{p q}+\sum_{p, q \in X: p<q} s_{p q} \geq \sum_{p \in P, q \in X} \sum_{j=1}^{d}\left(v_{p q}^{j}\right)^{2}+\sum_{p, q \in X: p<q} \sum_{j=1}^{d}\left(w_{p q}^{j}\right)^{2},
$$

which means that the lower bounds found using the relaxation $\left(R_{4}\right)$ are as good as the bound found using the relaxation $\left(R_{2}\right)$.

\subsection{Fifth Relaxation}

The fifth relaxation is derived from the third model $\left(M_{3}\right)$. It is defined as follows:

$$
\begin{aligned}
& \min \sum_{p \in P, q \in X} \sum_{j=1}^{d}\left(u_{p q}^{j}-\zeta_{j}^{p} y_{p q}\right)^{2}+ \\
& \sum_{p, q \in X: p<q}\left(\sum_{j=1}^{d}\left(w_{p q}^{j}\right)^{2}-\sum_{j=1}^{d} x_{j}^{p} w_{p q}^{j}-\sum_{j=1}^{d} x_{j}^{q} v_{p q}^{j}+\sum_{j=1}^{d}\left(v_{p q}^{j}\right)^{2}\right) \\
& \text { s.t. } \\
& \quad(2)-(8) \text { and }(36)-(45) .
\end{aligned}
$$

The objective function of the relaxation $\left(R_{5}\right)$ is smooth but not convex. 


\subsection{Sixth Relaxation}

The sixth relaxation is derived from the fourth model $\left(M_{4}\right)$ and it is defined as follows:

$$
\min \sum_{p \in P, q \in X} \sum_{j=1}^{d}\left(u_{p q}^{j}-\zeta_{j}^{p} y_{p q}\right)^{2}+\sum_{p, q \in X: p<q} \sum_{j=1}^{d}\left(w_{p q}^{j}-v_{p q}^{j}\right)^{2}
$$

s.t.

$$
\text { (2) - (8) and (48) - (57). }
$$

The objective function in the relaxation $\left(R_{6}\right)$ is smooth and convex.

\section{Preliminary Computational Results}

The main goal of these preliminary computational results is to highlight the main features, from a computational point of view, of the proposed relaxations. At some points, comparisons will be made with the solutions found using Smith's exact algorithm (see [18]). The solution time of all the presented experiences was fixed to 3 hours.

\subsection{The Instances}

Two sets of instances are considered. The first one contains five instances related to the five platonic solids. In each instance, the coordinates of the terminals are the coordinates of the vertices of the corresponding platonic solid in $\mathbb{R}^{3}$. These instances are named: Tetra, Octa, Cube, Icosa and Dodec. Recall that: the tetrahedron has 4 vertices; the octahedron has 6 vertices; the cube has 8 vertices; the icosahedron has 12 vertices; and the dodecahedron has 20 vertices.

The second set of instances is a subset of Smith's instances representing simplicies and octahedra in $\mathbb{R}^{d}$. The dimension of the simplicies varies from 3 to 8 and the dimension of the octahedra varies from 4 to 6 . These instances are named NSimp-x and NOcta-x, where $\mathrm{x}$ indicates the value of the dimension $d$.

All the coordinates of these instances are scaled in ordered to fulfill the assumption 1 .

\subsection{The Results}

The following Tables 1 and 2 sum up, for each instance and every relaxation the following items: the lower bound value (denoted $\mathrm{lb}$ ), the upper bound value (denoted ub), the gap between the upper and lower bounds (denoted gap and defined as being: $\left.\frac{u b-l b}{u b} \times 100\right)$, and the gap between the upper bound ub and the length of the best feasible solution found using Smith's algorithm (this gap is denoted gap* and defined as been $\frac{u b-\text { Best.sol }}{u b} \times 100$ ). For a relaxation $R$ and an instance I: $I \mathrm{~b}$ is the value of the best solution found after solving I using $R$ (the time limit being 3 hours); ub is the $\|\cdot\|_{2}$-length of this best solution ${ }^{1}$.

\footnotetext{
${ }^{1}$ Remember that the value of an optimal feasible solution of any relaxation is always a lower bound of its $\|.\|_{2}$-length, see the proposition 3
} 
Finally, in the column Best sol were reported the values of the best feasible solutions found using Smith's enumeration algorithm (see [18]).

The solver Artelys-Knitro (see https://www.artelys.com/) is used to solve the relaxations $R_{1}$ and $R_{5}$. The other relaxations are solved using the Ilog-Cplex (see https://www.ibm. com/) solver. These solvers are used via the ampl (see https://ampl.com/) modeling language. As stated before, the time limit was fixed to 3 hours. All of our instances are solved on a 8Gb Ram and 2.7 $\mathrm{GHz} \times 4$ Inter-Core processor running under Linux Ubuntu 16.04.5 LTS 64 Bits.

Few comments on the results reported in Tables 1 and 2 are in order. First notice that, the relative gap between the upper and lower bounds is very important! Indeed, the value of gap is between $83 \%$ and $95 \%$ for all the relaxations except $R_{4}$. The value of gap for the relaxation $R_{4}$ is between $35 \%$ and $67 \%$. This may indicate that the relaxation $R_{4}$ may perform well. This is not the case if one compares the values of gap*.

\begin{tabular}{|c|c|c|c|c|c|c|c|c|c|c|c|c|c|}
\hline \multirow[b]{2}{*}{ Instance } & \multicolumn{4}{|c|}{$R_{1}$} & \multicolumn{4}{|c|}{$R_{2}$} & \multicolumn{4}{|c|}{$R_{3}$} & \multirow[b]{2}{*}{ Best sol. } \\
\hline & $\mathrm{lb}$ & $\mathrm{ub}$ & $\begin{array}{l}\text { gap } \\
(\%)\end{array}$ & $\begin{array}{c}\text { gap* } \\
(\%)\end{array}$ & lb & $\mathrm{ub}$ & $\begin{array}{l}\text { gap } \\
(\%)\end{array}$ & $\begin{array}{l}\text { gap* } \\
(\%)\end{array}$ & $1 \mathrm{~b}$ & $\mathrm{ub}$ & $\begin{array}{l}\text { gap } \\
(\%)\end{array}$ & $\begin{array}{c}\text { gap* } \\
(\%)\end{array}$ & \\
\hline Tetra & 0.138889 & 0.824958 & 83.16 & 1.44 & 0.138889 & 0.824958 & 83.16 & 1.44 & 0.138889 & 0.824956 & 83.16 & 1.44 & 0.8130525127 \\
\hline Octa & 0.107071 & 0.969439 & 88.96 & 1.39 & 0.107071 & 0.969439 & 88.96 & 1.39 & 0.107069 & 0.969435 & 88.96 & 1.39 & 0.9560044889 \\
\hline Cube & 0.11358 & 1.202142 & 90.55 & 0.81 & 0.113469 & 1.204798 & 90.58 & 1.02 & 0.113468 & 1.204795 & 90.58 & 1.02 & 1.1924500991 \\
\hline Icosa & 0.214883 & 2.009197 & 89.31 & 19.09 & 0.13004 & 1.633776 & 92.04 & 0.50 & 0.131442 & 1.642929 & 92.00 & 1.05 & 1.6256392494 \\
\hline Dodeca & 0.322825 & 3.238779 & 90.03 & 31.73 & 0.146715 & 2.305906 & 93.64 & 4.12 & 0.192682 & 2.592707 & 92.57 & 14.72 & 2.2110017813 \\
\hline NOcta-4 & 0.07568 & 0.974522 & 92.23 & 2.39 & 0.075348 & 0.972001 & 92.25 & 2.14 & 0.075344 & 0.972004 & 92.25 & 2.14 & 0.9512411857 \\
\hline NOcta-5 & 0.072482 & 1.027447 & 92.95 & 7.62 & 0.05863 & 0.978012 & 94.01 & 2.95 & 0.05863 & 0.978011 & 94.01 & 2.95 & 0.9491744441 \\
\hline NOcta-6 & 0.052298 & 1.020536 & 94.88 & 7.07 & 0.04819 & 0.984459 & 95.10 & 3.66 & 0.048428 & 0.987845 & 95.10 & 3.99 & 0.9484338012 \\
\hline NSimp-3 & 0.078125 & 0.618718 & 87.37 & 1.44 & 0.078125 & 0.618718 & 87.37 & 1.44 & 0.078125 & 0.61871 & 87.37 & 1.44 & 0.6097893868 \\
\hline NSimp-4 & 0.060952 & 0.64171 & 90.50 & 2.29 & 0.060952 & 0.64171 & 90.50 & 2.29 & 0.060952 & 0.641716 & 90.50 & 2.29 & 0.6269985606 \\
\hline NSimp-5 & 0.05 & 0.656676 & 92.39 & 2.98 & 0.05 & 0.656676 & 92.39 & 2.98 & 0.049995 & 0.656683 & 92.39 & 2.98 & 0.6371368899 \\
\hline NSimp-6 & 0.043132 & 0.664619 & 93.51 & 3.09 & 0.042375 & 0.667347 & 93.65 & 3.49 & 0.042355 & 0.66732 & 93.65 & 3.48 & 0.6440799752 \\
\hline NSimp-7 & 0.036762 & 0.675356 & 94.56 & 3.96 & 0.036762 & 0.675356 & 94.56 & 3.96 & 0.036712 & 0.675357 & 94.56 & 3.96 & 0.6486057997 \\
\hline NSimp-8 & 0.032459 & 0.681588 & 95.24 & 4.31 & 0.032459 & 0.681588 & 95.24 & 4.31 & 0.032407 & 0.681696 & 95.25 & 4.32 & 0.6522373981 \\
\hline
\end{tabular}

Table 1: Lower and upper bounds obtained using the relaxations $R_{1}, R_{2}$ and $R_{3}$.

\begin{tabular}{|c|c|c|c|c|c|c|c|c|c|c|c|c|c|}
\hline \multirow[b]{2}{*}{ Instance } & \multicolumn{4}{|c|}{$R_{4}$} & \multicolumn{4}{|c|}{$R_{5}$} & \multicolumn{4}{|c|}{$R_{6}$} & \multirow[b]{2}{*}{ Best sol. } \\
\hline & $1 \mathrm{~b}$ & $\mathrm{ub}$ & $\begin{array}{l}\text { gap } \\
(\%)\end{array}$ & $\underset{(\%)}{\text { gap* }}$ & $1 \mathrm{~b}$ & $\mathrm{ub}$ & $\begin{array}{l}\text { gap } \\
(\%)\end{array}$ & $\begin{array}{c}\text { gap* } \\
(\%)\end{array}$ & $1 \mathrm{~b}$ & $\mathrm{ub}$ & $\begin{array}{l}\text { gap } \\
(\%)\end{array}$ & $\begin{array}{c}\text { gap* } \\
(\%)\end{array}$ & \\
\hline Tetra & 0.58317 & 0.909546 & 35.88 & 10.61 & 0.138889 & 0.824958 & 83.16 & 1.44 & 0.138889 & 0.824958 & 83.16 & 1.44 & 0.8130525127 \\
\hline Octa & 0.666667 & 1.101728 & 39.49 & 13.23 & 0.107071 & 0.969439 & 88.96 & 1.39 & 0.107071 & 0.969439 & 88.96 & 1.39 & 0.9560044889 \\
\hline Cube & 0.7698 & 1.333333 & 42.26 & 10.57 & 0.115929 & 1.213361 & 90.45 & 1.72 & 0.113469 & 1.204798 & 90.58 & 1.02 & 1.1924500991 \\
\hline Icosa & 1.107124 & 1.727303 & 35.90 & 5.89 & 0.254982 & 2.116967 & 87.96 & 23.21 & 0.13004 & 1.633776 & 92.04 & 0.50 & 1.6256392494 \\
\hline Dodeca & 1.559627 & 2.521275 & 38.14 & 12.31 & na & na & na & na & 0.155806 & 2.35431 & 93.38 & 6.09 & 2.2110017813 \\
\hline NOcta-4 & 0.625 & 1.25 & 50.00 & 23.90 & 0.075348 & 0.972001 & 92.25 & 2.14 & 0.075348 & 0.972001 & 92.25 & 2.14 & 0.9512411857 \\
\hline NOcta-5 & 0.6 & 1.341641 & 55.28 & 29.25 & na & na & na & na & 0.05863 & 0.978012 & 94.01 & 2.95 & 0.9491744441 \\
\hline NOcta-6 & 0.583333 & 1.342596 & 56.55 & 29.36 & na & na & na & na & 0.04819 & 0.984459 & 95.10 & 3.66 & 0.9484338012 \\
\hline NSimp-3 & 0.353553 & 0.707107 & 50.00 & 13.76 & 0.078125 & 0.618718 & 87.37 & 1.44 & 0.078125 & 0.618718 & 87.37 & 1.44 & 0.6097893868 \\
\hline NSimp-4 & 0.353553 & 0.790569 & 55.28 & 20.69 & 0.060952 & 0.64171 & 90.50 & 2.29 & 0.060952 & 0.64171 & 90.50 & 2.29 & 0.6269985606 \\
\hline NSimp-5 & 0.353553 & 0.866025 & 59.18 & 26.43 & 0.05 & 0.656676 & 92.39 & 2.98 & 0.05 & 0.656676 & 92.39 & 2.98 & 0.6371368899 \\
\hline NSimp-6 & 0.353553 & 0.935414 & 62.20 & 31.14 & 0.042375 & 0.667347 & 93.65 & 3.49 & 0.042375 & 0.667347 & 93.65 & 3.44 & 0.6440799752 \\
\hline NSimp-7 & 0.353553 & 1 & 64.64 & 35.14 & 0.036762 & 0.675356 & 94.56 & 3.96 & 0.036762 & 0.675356 & 94.56 & 3.96 & 0.6486057997 \\
\hline NSimp-8 & 0.353553 & 1.06066 & 66.67 & 38.51 & na & na & na & na & 0.032459 & 0.681588 & 95.24 & 4.31 & 0.6522373981 \\
\hline
\end{tabular}

Table 2: Lower and upper bounds obtained using the relaxations $R_{4}, R_{5}$ and $R_{6}$.

Considering all instances, except Icosa and Dodec, the value of gap* of all the relaxations except the relaxation $R_{4}$ is at most $4 \%$ while the minimum value of gap* for $R_{4}$ is $10.5 \%$. So, using the relaxation $R_{4}$ is not a good choice if one 
is interested in finding only a feasible solution to the Euclidean Steiner tree problem.

The relaxation $R_{5}$ is the most difficult to solve since several instances were not solved. Recall that this relaxation consists of optimizing a non convex objective function over a polyhedron.

The two instances Icosa and Dodec are the most difficult to solve, especially Dodec. The best upper bound for these two instances are found using the relaxation $R_{2}$, a gap* equal to $0.5 \%$ for Icosa and around $4 \%$ for Dodec. Also, the feasible solution found using the relaxation $R_{6}$ has a gap* equal to $0.5 \%$.

Regarding Smith's instances, the value of gap* is at most $5 \%$ for all relaxations except $R_{4}$ for which the minimum gap* is equal to $13.76 \%$. The value of gap* increases slightly ( $1 \%$ in average) with the dimension $d$. Because of the size of the relaxation (number of variables and constraints) which increases with the dimension $d$.

Finally, depicted below are the feasible solutions of the platonic instances found using relaxations $R_{2}$ and $R_{6}$. As indicated in each picture, the left image is a feasible solution found using $R_{2}$ and the right image is the feasible solution found using $R_{6}$. Notice that, for each considered instance, the topologies are different.
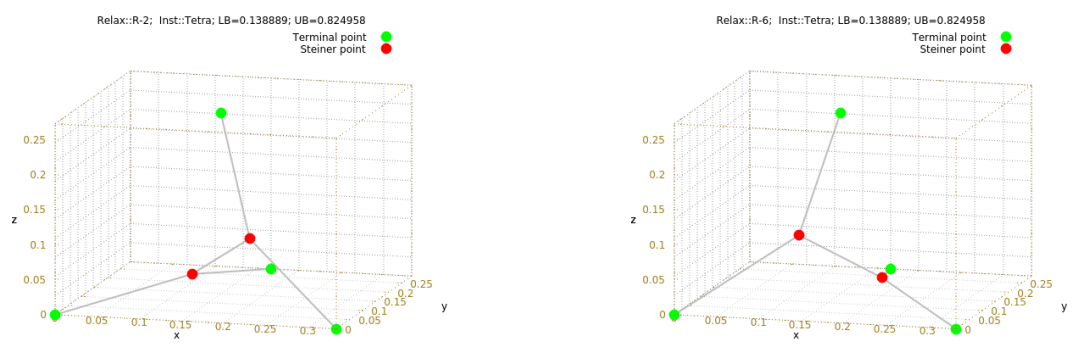

Figure 1: Tetrahedron instance.
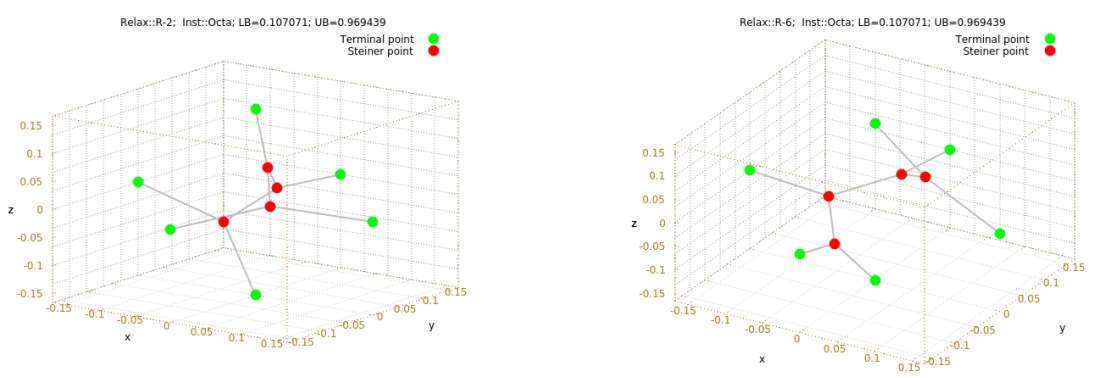

Figure 2: Octahedron instance. 

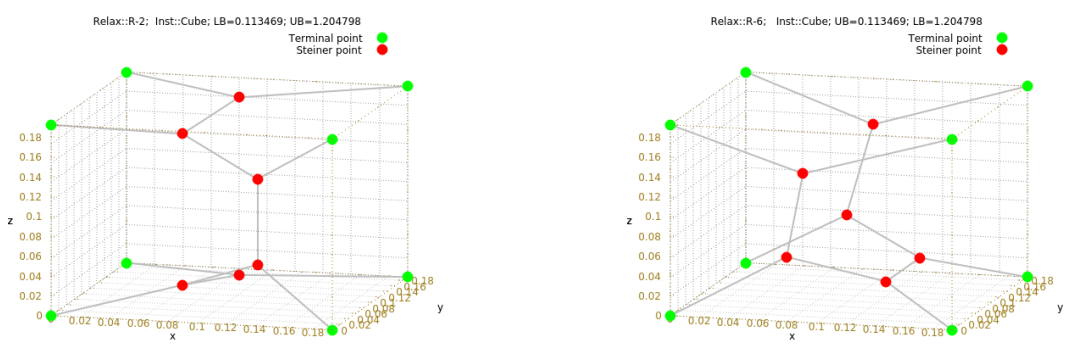

Figure 3: Cube instance.
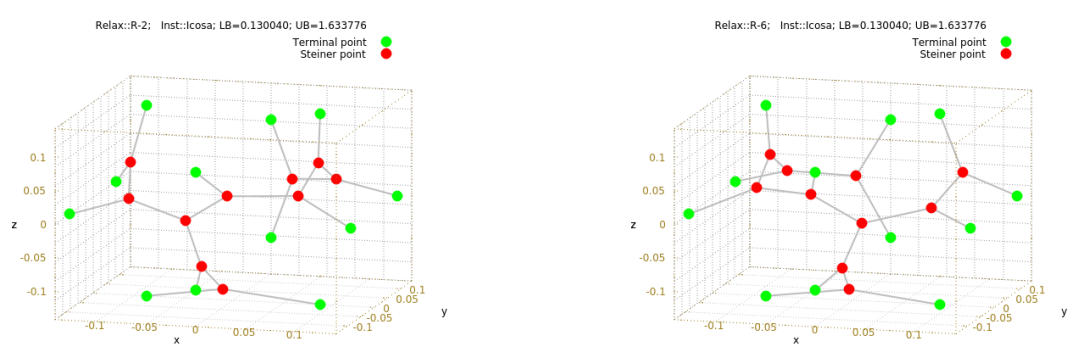

Figure 4: Icosahedron instance.
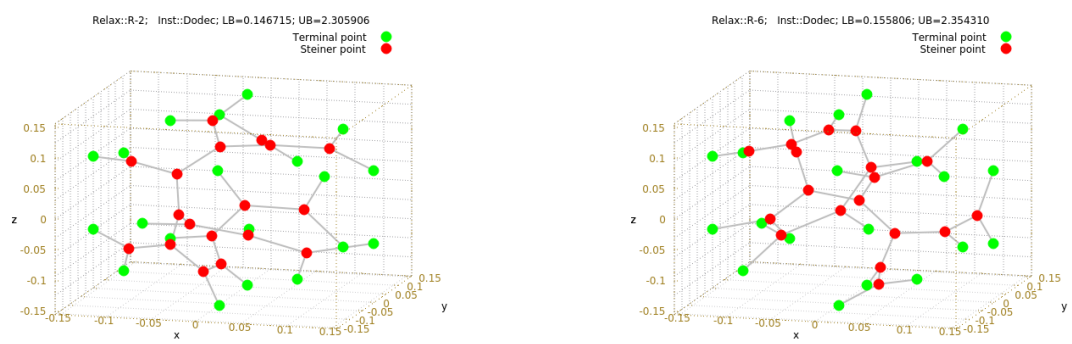

Figure 5: Dodecahedron instance.

\section{Conclusion}

In this work, new models for the Euclidean Steiner tree problem were presented. These models are shown to be theoretically equivalent. Six linear versus nonlinear mixed integer relaxations were derived from these models: $R_{1}$ is a nonlinear and non convex mixed integer relaxation; $R_{2}$ and $R_{6}$ are a convex quadratic 
mixed integer relaxations; $R_{3}$ and $R_{4}$ are mixed integer linear relaxations; and, finally, $R_{5}$ is a non convex quadratic relaxation. Solving these relaxations permits, for a given instance, the computation of the lower and upper bounds of the length of the minimum Steiner tree. The upper bound found with any relaxation corresponds to the length of a feasible Steiner tree. The preliminary computational results show that even if the values of the lower bounds are very poor, the values of the upper bounds are rather tight. So, one can for instance investigate the efficiency of an ad hoc algorithm to solve, exactly or approximately (generating good feasible solutions), both relaxations $R_{2}$ and $R_{6}$.

\section{Acknowledgements}

We are grateful to the referees for their helpful remarks.

This work was partially supported by the National Council for Scientific and Technological Development - CNPq, under grant 302578/2014-5, COPPETEC Foundation, and by CAPES-MEC.

\section{References}

[1] M. Brazil, R.L. Graham, D.A. Thomas, and M. Zachariasen. On the history of the euclidean steiner tree problem. Archive for History of Exact Sciences, 68:327-354, 2014.

[2] M. Brazil, D. A. Thomas, B. K. Nielson, P. Winter, C. Wulff-Nilsen, and M. Zachariasen. A novel approach to phylogenetic trees: $d$-dimensional geoemtric steiner trees. Networks, 53(2):104-111, 2009.

[3] L. L. Cavalli-Sforza and A. W. F. Edwards. Phylogenetic analysis: Models and estimation procedures. Evolution, 21:550-570, 1967.

[4] Warm D. M., P. Winter, and M. Zachariasen. Exact algorithms for plan steiner tree problems: A computational study. Technical report, 1998.

[5] Warm D. M. and M. Zachariasen. Large euclidean steiner minimum trees in an hour. In ISMP, 1997.

[6] C. D'Ambrosio, M.H.C Fampa, J. Lee, and S. Vigerske. Formulation of the euclidean steiner tree problem in n-space: missing proofs. Optimization Letters, accepted.

[7] M. Fampa and K. Anstreicher. An improved algorithm for computing steiner minimal trees in eucidean $d$-space. Discrete Optimization, (5):530540, 2008.

[8] M.H.C Fampa, J. Lee, and N. Maculan. An overview of exact algorithms for the euclidean steiner tree problem in $n$-space. International Transactions in Operational Research (ITOR), 23(4):861-874, 2016. 
[9] M.H.C Fampa and N. Maculan. A new relaxation in conic form for the euclidean steiner problem in $\mathbb{R}^{n}$. RAIRO - Operations Research, 35(4):283394, 2001.

[10] M.H.C Fampa and N. Maculan. Using a conic formulation for finding steiner minimal trees. Numerical Algorithms, 35(4):315-330, 2004.

[11] R. Fonseca, M. Brazil, P. Winter, and M. Zachariasen. Faster exact algorithms for computing steiner trees in higher dimensional euclidean spaces. Presented at the 11th DIMACS Implementation Challenge Workshop, http://dimacs11.zib.de/workshop/FonsecaBrazilWinterZachariasen.pdf, 2014.

[12] M. R. Garey, R. L. Graham, and D. S. Johnson. Complexity of computing steiner minimal trees. SIAM J. Appl. Math., 32(4):835-859, June 1977.

[13] M. R. Garey and D. S. Johnson. Computers and Intratability: A Guide to the Theory of NP-Completeness. W.H. Freeman and Company, San Francisco, CA, USA, 1979.

[14] E. N. Gilbert and H. O. Pollak. Steiner minimal trees. SIAM Journal on Applied Mathematics, 16(1):1-29, 1968.

[15] N. Maculan, Ph. Michelon, and A.E. Xavier. The euclidean steiner tree problem in $\mathbb{R}^{n}$ : a mathematical programming formulation. Annals of Operations Research, 96:209-220, 2000.

[16] F. Montenegro, J.R.A Torreão, and N. Maculan. Microcanonical optimization for the euclidean steiner problem in $\mathbb{R}^{n}$ with application to phylogenetic inference. Physical Review E, 68:056702-1- 056702-5, 2003.

[17] J. M. Smith, Y. Jang, and M. K. Kim. Steiner minimal trees, twist angles, and the protein folding problem. Proteins: Structures, Functions, and Bioinformatics, 66(4):889-902, 2007.

[18] W. D. Smith. How to find steiner minimal trees in euclidean $d$-space. Algorithmica, 7(2-3):137-177, 1992.

[19] C. Stanton and J. M. Smith. Steiner trees and 3-d macromolecular conformation. Informs journal on computing, 16:470-485, 2004.

[20] M. Zachariasen. Large Euclidean Steiner Minimum Trees in an Hour. PhD thesis, University of Copenhagen, 1998. 